\title{
Some control observation problems and their differential algebraic partial solutions
}

\author{
Sette Diop \\ Laboratoire des Signaux \& Systèmes, CNRS-CentraleSupélec-Univ Paris-Sud, \\ Université Paris-Saclay, Plateau de Moulon 3 rue Joliot Curie 91192 Gif sur Yvette \\ cedex France \\ Diop@L2S.CentraleSupelec.fr
}

\begin{abstract}
Observation problems in control systems literature generally refer to problems of estimation of state variables (or identification of model parameters) from two sources of information: dynamic models of systems consisting in first order differential equations relating all system quantities, and online measurements of some of these quantities. For nonlinear systems the classical approach stems from the work of R. E. Kalman on the distinguishability of state space points given the knowledge of time histories of the output and input. In the differential algebraic approach observability is rather viewed as the ability to recover trajectories. This approach turns out to be a particularly suitable language to describe observability and related questions as structural properties of control systems. The present paper is an update on the latter approach initiated in the late eighties and early nineties by J. F. Pommaret, M. Fliess, S. T. Glad and the author.
\end{abstract}

Keywords: Control observation problems; State estimation; Differential algebraic decision methods; Differential algebraic geometry

\section{Introduction}

Observation problems in control systems literature generally refer to problems of estimation of state variables $x$ from two sources of information: online measurements of external variables $u$ and $y$, and first order dynamic models

$$
\left\{\begin{array}{l}
\dot{x}=f(t, u, x) \\
y=h(t, u, x)
\end{array}\right.
$$

relating $x$ to $u$ and $y$. See for instance $[1,2]$.

By using tools from differential algebraic geometry

- dynamic models are allowed to be implicit and of arbitrary order but restricted to be polynomial in variables and their derivatives,

- and may be considered more general situations of estimating one subset of system variables with respect to another subset of the system variables. 
Specifically, given a dynamic system described by algebraic differential equations

$$
\left\{\begin{array}{l}
P_{i}(w, z, \zeta)=0 \quad(i=1,2, \cdots), \\
Q(w, z, \zeta) \neq 0
\end{array}\right.
$$

one observation problem consists of the online estimation of $z(t) \in \mathbb{R}^{\nu}$ from the knowledge of the $P_{i}$ 's and $Q$ and time histories $\left(\left[t_{0}, t\right] \ni \tau \longmapsto w(\tau) \in \mathbb{R}^{\mu}\right)$ of $w$. Here the $P_{i}$ 's and $Q$ are differential polynomials in $w, z$ and $\zeta$, and $\nu, \mu$ are natural integers. This problem is under investigation since the pioneering work of R. E. Kalman in the late fifties addressing its linear context. A complete nonlinear answer is still lacking. A general approach consists of a two part theory: one of observability, that is, derivation of conditions on the $P_{i}$ 's and $Q$ guaranteeing the ability to some how estimate $z$ from the supposedly known data, and the other part of the theory, the observer design, searches algorithms for such an estimation of $z$.

Though central the previous observation problem (observability and observer design) is not the only one. For instance, closely related to it, are two problems of robustness with respect to model and measurements uncertainties. Another observation problem with important practical application consists of determining subsets $w$ of systems variables which make a given subset $z$ observable.

Starting from the mid eighties (see [3-6]) differential algebra and differential algebraic decision methods have been shown to provide a quite consistent language to describe some of these observation problems along with some of their solutions.

An account of this is proposed here. Some of the many open problems will be described.

Reviewers of the present paper suggested appending to it materials of differential algebra which are invoked throughout. Such an account has been done already in [6] and would double the space of the present paper. These are the reasons why we prefer referring the reader to the appendix of [6] instead of duplicating here those materials of differential algebra, differential algebraic geometry and and differential algebraic decision methods.

\section{The differential algebraic approach}

A thorough introduction to the differential algebraic approach is available in [6]. For the sake of completeness the following definition is recalled from there.

A (differential) (algebraic) system $\mathcal{X}$ with $s$ variables, and with coefficients in a differential field $\mathbf{k}$ is a proper differential quasi-affine variety $\mathcal{X} \subseteq \overline{\mathbf{k}}^{s}$ defined over $\mathbf{k}$ where $\overline{\mathbf{k}}$ is a differential closure of $\mathbf{k}$. In observation problems, the system variable is partitioned into the data, or observations, $w=w_{1}, \ldots, w_{\mu}$, the variable being observed (or estimated) $z=z_{1}, \ldots, z_{n}$ and the remaining variables, $\zeta$. In the classical observation problem, the data consist exclusively of $(u$, $y$ ), the control $u$ and the measurements $y$. When the variable $\zeta$ is present, the projection $\mathcal{X}_{w, z}$ of $\mathcal{X}$ along the variable $\zeta$ is considered. It is the set of elements $(\bar{w}, \bar{z}) \in \overline{\mathbf{k}}^{\mu} \times \overline{\mathbf{k}}^{n}$ such that there is at least $\bar{\zeta}$ such that $(\bar{w}, \bar{z}, \bar{\zeta}) \in \mathcal{X}$. 
In terms of equations, previously defined systems are those described by

$$
\left\{\begin{array}{l}
P_{i}(w, z, \zeta)=0, \quad i=1,2, \ldots \\
Q(w, z, \zeta) \neq 0
\end{array}\right.
$$

where the $P_{i}$ 's and $Q$ are finitely many polynomials in $w, z, \zeta$ and their derivatives.

For a system $\mathcal{X}$ the variable $z$ is said to be (algebraically) observable with respect to $w$ if the projection map $\pi: \mathcal{X}_{w, z} \rightarrow \mathcal{X}_{w}$ (sending every trajectory $(\bar{w}, \bar{z})$ of $\mathcal{X}_{w, z}$ onto the corresponding observation $\left.\bar{w}\right)$ is generically finite. If $z$ is observable with respect to $w$ then the degree of $\pi$ is called the observability degree of $z$ with respect to $w$, and is denoted by $\mathrm{d}_{w}^{\circ} z$. The variable $z$ is said to be rationally observable with respect to $w$ if it is observable with respect to $w$ with observability degree one. State systems of the form (1) are said to be observable if $x$ is observable with respect to $(u, y)$.

It was first proved in [5] (see [6] for more details) that the previous definition has a differential algebraic translation, namely: $z$ is observable with respect to $w$ iff $z$ is algebraic over $\mathbf{k}\langle w\rangle$, that is, for each component, $z_{i}$ of $z$ there is a polynomial equation

$$
H_{i}\left(z_{i}, w, \dot{w}, \ldots\right)=0
$$

in $z_{i}$, and finitely many time derivatives of the data $w$, with coefficients in $\mathbf{k}$.

The reader is referred to [6] more details on differential algebraic geometry terms or notations used here without explanations.

\section{How does it compare to the classical theory?}

Formal definitions of observability can be found in $[1,2]$ for instance.

For linear state systems

$$
\left\{\begin{array}{l}
\dot{x}=F x+G u \\
y=H x+E u
\end{array}\right.
$$

the answer to the question is that algebraically observability of $x$ with respect to $(u, y)$ is equivalent to the classical Kalman definition of observability of system (5). The proof of this is as follows.

As is well known system (5) is observable in the classical sense iff $\operatorname{rk}_{\mathbb{R}} \mathcal{O}(F, H)=$ $n$, where $n$ is the number of components of the state, $x$, and where

$$
\mathcal{O}(F, H)=\left(\begin{array}{c}
H \\
H F \\
\vdots \\
H F^{n-1}
\end{array}\right) .
$$


Now the following equalities

$$
\begin{array}{rlrl}
H x & =y-E u & & =z_{0}, \\
H F x & =\dot{z}_{0}-H G u & & =z_{1}, \\
H F^{2} x & =\dot{z}_{1}-H F G u & & =z_{2}, \\
\vdots & & \\
H F^{n-1} x & =\dot{z}_{n-2}-H F^{n-2} G u & =z_{n-1},
\end{array}
$$

result from the equations of system (5). The reader will notice that they are written such that only supposedly differentiable quantities are differentiated. They may be rewritten as

$$
\mathcal{O}(F, H) x=\left(\begin{array}{c}
z_{0} \\
z_{1} \\
z_{2} \\
\vdots \\
z_{n-1}
\end{array}\right)
$$

Therefore if system (5) is observable in the classical sense then it is so in the algebraic sense. The converse follows from Corollary 1 below.

The equivalence between the algebraic and the classical definitions of observability for the class of systems (5) was first mentioned in $\S 5.1 .2$ of [7] but without a complete proof.

It is worth emphasizing the fact that the algebraic definition of systems applies without any change to so-called implicit or descriptor linear systems

$$
\left\{\begin{aligned}
M \dot{x} & =F x+G u, \\
y & =H x+E u,
\end{aligned}\right.
$$

where the matrix $M$ is singular. Compare to [8] and references therein.

The largest class of systems where algebraic and classical observability may be compared is the class of rational state systems

$$
\left\{\begin{array}{l}
\dot{x}_{i}=\frac{p_{i}(u, x)}{q_{i}(u, x)}(1 \leq i \leq n), \\
y_{j}=\frac{f_{j}(u, x)}{g_{i}(u, x)}(1 \leq j \leq p),
\end{array}\right.
$$

where $u$ stands for $u_{1}, u_{2}, \ldots, u_{m}$, and $p_{i}, q_{i}, f_{i}$ and $g_{i}$ are differential polynomials of order zero in $x$ with coefficients in $\mathbf{k}=\mathbb{R}$, the algebraic observability (of $x$ with respect to $(u, y))$ is equivalent to the generic local observability of the system as defined in [1]. This was first obtained in [9].

\section{Partial answers to some observation problems}

Among all benefits of the differential algebraic approach to observation problems, application of decision methods is perhaps the most appealing. 


\subsection{Computing}

One of the most used estimation algorithms, the Kalman filter (or its extended form), is often applied in practice without prior asserting the validity of its conditions. The reason of this is that there is no systematic method for such a verification.

The differential algebraic theory of observability is constructive in principle.

Most of the decision methods which may be used are already described in [6]. This is the case for general polynomial systems

$$
P_{i}(w, z, \zeta)=0, \quad i=1,2, \ldots
$$

with constant coefficients. For rational state systems (8) the observability test is formally similar to the Jacobian rank condition which is found in [1].

For polynomial state systems with nonconstant coefficients let $\mathbf{k}$ be an ordinary differential field (not necessarily of constants). Let $\mathcal{X}$ be

$$
\left\{\begin{array}{l}
\dot{x}_{i}=f_{i}(u, x)(1 \leq i \leq n), \\
y_{j}=h_{j}(u, x)(1 \leq j \leq p),
\end{array}\right.
$$

where the $f_{i}$ 's and $h_{j}$ 's are (nondifferential) polynomials in their arguments with coefficients in $\mathbf{k}$. Let

$$
\begin{aligned}
P_{i}(U, X, Y) & =X_{i}^{(1)}-f_{i}(U, X) \quad(1 \leq i \leq n) ; \\
P_{n+j}(U, X, Y) & =h_{j}(U, X)-Y_{j} \quad(1 \leq j \leq p)
\end{aligned}
$$

be the differential polynomials defining $\mathcal{X}$. Let $\sigma: \mathbf{k}\{U, Y\} \rightarrow \mathbf{k}\{u, y\}$ be the substitution map which sends $U$ to $u$ and $Y$ to $y$, where $\mathbf{k}\{u, y\}$ is the differential $\mathbf{k}$-subalgebra of $\mathbf{k}\{u, x, y\}$ generated over $\mathbf{k}$ by $u$ and $y$. Let $P$ be in $\mathbf{k}\{U, X, Y\}$ and $P^{\sigma}$ denote the element of $\mathbf{k}\{u, y\}\{X\}$ obtained by regarding $P$ as a differential polynomial in $X$ with coefficients in $\mathbf{k}\{U, Y\}$ and by applying $\sigma$ to each of these coefficients, and let $\mathbf{I}(\mathcal{X})^{\sigma}$ stand for the differential ideal of $\mathbf{k}\{u, y\}\{X\}$ consisting of $P^{\sigma}(P \in \mathbf{I}(\mathcal{X}))$. The ideal of definition, $\mathfrak{a}$, of $\mathbf{k}\langle u, y\rangle(x)$ over $\mathbf{k}\langle u, y\rangle$ is equal to $\mathbf{I}(\mathcal{X})^{\sigma} \cap \mathbf{k}\langle u, y\rangle\left[\left(X_{i}\right)_{1 \leq i \leq n}\right]$. Note that the set $\mathcal{A}$ consisting of the $P_{i}(1 \leq i \leq n)$ form an autoreduced set with respect to any ranking of $\mathbf{k}\{U, X, Y\}$ such that $U, Y$ and their derivatives all are lower than $X$.

Let us now inductively define some polynomials which will turn out to be generators of the ideal $\mathbf{I}(\mathcal{X})^{\sigma} \cap \mathbf{k}\langle u, y\rangle\left[\left(X_{i}\right)_{1 \leq i \leq n}\right]$ of $\mathbf{k}\langle u, y\rangle\left[\left(X_{i}\right)_{1 \leq i \leq n}\right]$.

Starting with

$$
Q_{i}(U, X, Y)=P_{n+i}(U, X, Y) \quad(1 \leq i \leq p),
$$

then let $Q_{p+i}$ be the remainder of the derivative of $Q_{i}(1 \leq j \leq n)$ with respect to the previously mentioned autoreduced set, $\mathcal{A}$. The polynomial $Q_{p+i}$ is merely the derivative of $Q_{i}$ in which $X_{j}^{(1)}$ is eliminated by substituting $P_{j}+f_{j}$ for $X_{j}^{(1)}(1 \leq j \leq n)$ (The linear combination of $P_{j}(1 \leq j \leq n)$ which appears 
reduces to zero when the remainder is taken, so that it can be ignored.) Explicitly, $Q_{p+i}$ is as follows

$$
\begin{aligned}
Q_{p+i}= & \sum_{1 \leq j \leq m} \frac{\partial Q_{i}}{\partial U_{j}} U_{j}^{(1)}+\sum_{1 \leq j \leq n} \frac{\partial Q_{i}}{\partial X_{j}} f_{j}-Y_{i}^{(1)} \\
& +Q_{i} \bullet \quad(1 \leq i \leq p),
\end{aligned}
$$

where the notations

$$
P_{\bullet} \equiv P_{(1)}, \quad P_{(2)}, \quad \cdots
$$

for a differential polynomial $P$ stand for the differential polynomials obtained by replacing the coefficients of $P$ by their respective derivatives respectively at order 1,2 , etc.

Note that this formula is nothing but a counterpart of Lie derivatives: Authors usually consider the functions $h_{j}$ as free of $u$ and the functions $f_{i}$ and $h_{j}$ as with constant coefficients so that in the left hand side of the latter equation the first sum as well as the last term are absent.

This construction of $Q_{p+i}$ from $Q_{i}$ is iterated in order to get $Q_{2 p+i}(1 \leq i \leq p)$ as the remainder of the derivative of $Q_{p+i}(1 \leq i \leq p)$. And so on.

By their definition,

$$
Q_{i}^{\sigma} \in \mathbf{I}(\mathcal{X})^{\sigma} \bigcap \mathbf{k}\langle u, y\rangle\left[\left(X_{j}\right)_{1 \leq j \leq n}\right](i \in \mathbb{N}) .
$$

Conversely, let $P$

$$
P \in \mathbf{I}(\mathcal{X})^{\sigma} \bigcap \mathbf{k}\langle u, y\rangle\left[\left(X_{j}\right)_{1 \leq i \leq n}\right] .
$$

As an element of $\mathbf{I}(\mathcal{X}), P$ may easily be written in the form

$$
P=\sum_{1 \leq i \leq n, j \in \mathbb{N}} A_{i, j} P_{i}^{(j)}+\sum_{i \in \mathbb{N}} B_{i} Q_{i} .
$$

where $B_{i}(i \in \mathbb{N})$ are in

$$
\mathbf{I}(\mathcal{X}) \bigcap \mathbf{k}\{U, Y\}\left[\left(X_{i}\right)_{1 \leq i \leq n}\right] .
$$

Since the differential ideal of $\mathbf{k}\{U, X, Y\}$ generated by $P_{i}(1 \leq i \leq n)$ has no nonzero element in common with $\mathbf{I}(\mathcal{X}) \cap \mathbf{k}\{U, Y\}\left[\left(X_{j}\right)_{1 \leq i \leq n}\right]$ (this results from an obvious degree argument), the first sum in the previous equality must be zero.

This ends the proof that $Q_{i}^{\sigma}(i \in \mathbb{N})$ form a basis of $\mathfrak{a}$.

Lemma 1. A set of generators of the ideal of definition of $\mathbf{k}\langle u, y\rangle(x)$ over $\mathbf{k}\langle u, y\rangle$ is given by

$$
\begin{aligned}
& Q_{1}(u, X, y), Q_{2}(u, X, y), \ldots, Q_{p}(u, X, y), \\
& Q_{p+1}(u, X, y), Q_{p+2}(u, X, y), \ldots, Q_{2 p}(u, X, y),
\end{aligned}
$$


In addition, it comes from the Hilbert basis theorem that only finitely many $Q_{i}$ suffice to generate the ideal $\mathfrak{a}$. That is, there is some $\mu$ in $\mathbb{N}$ such that the first $\mu$ rows of the previous list of $Q_{i}(u, X, y)$ generate the ideal of definition of $\mathbf{k}\langle u, y\rangle(x)$ over $\mathbf{k}\langle u, y\rangle$. According to Theorem 16 of [6], the observability of $\mathcal{X}$ is equivalent to the fact that the $\mathbf{k}\langle u, y\rangle(x)$-matrix

$$
\left[\frac{\partial Q_{i}}{\partial X_{j}}(u, x, y)\right]_{\substack{1 \leq i \leq \mu p \\ 1 \leq j \leq n}}^{\substack{1 \\ \text { ind }}}
$$

is of rank $n$.

Now it is a basic fact that the above rank is equal to the rank of the first $n p$ rows.

Corollary 1. If $\mathcal{X}$ possesses a state description as above, then $\mathcal{X}$ is observable if, and only if, the following $\mathbf{k}\langle u, y\rangle(x)$-matrix

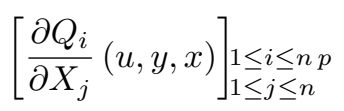

(which is formally the counterpart of the matrix of Lie derivatives which appears in the Hermann-Krener observability Jacobian rank condition) is of rank $n$.

The main difference between this rank condition and the one in [1] is that the rank is not over $\mathbf{k}$ (which is usually $\mathbb{R}$ ) but over a much bigger field (and, here the rank condition is a necessary and sufficient condition).

For arbitrary systems, observability tests resort on decision methods such as characteristic set of the defining differential ideal of $\mathcal{X}$. See [6] for more details. Very promising, Thomas decomposition was also proposed as decision methods for the same tests, see $[10,11]$.

\section{$5 \quad$ Regular observability}

The notion of regular observability refers to the classical one of universal inputs as thoroughly treated in [12]. Bad inputs (as opposed to universal ones) are supposed to occlude the functioning of online estimation schemes when they happened to be applied to a system. The present differential algebraic approach has brought a new light to this notion of singularity of the observability property. Here is an abstract of the result which may be found in [6] in more details.

Let $\mathcal{X}$ be a system with variables $w, z$, and $\zeta$, and with coefficients in $\mathbf{k}$. It is a matter of fact that, when $z$ is observable with respect to $w$ then for special observations $\bar{w}, \pi^{-1}(\bar{w})$ may contain infinitely many elements, leading to 
a singularity of the generic notion of observability. Here $\pi$ is the projection map of $\S 2$. An example of such situations is the following

$$
\left\{\begin{array}{l}
\dot{x}_{1}=x_{1} x_{2}, \\
\dot{x}_{2}=u+x_{2}, \\
y=x_{1} .
\end{array}\right.
$$

$x$ is observable with respect to $u, y$ since

$$
x_{1}=y \quad \text { and } \quad x_{2}=\frac{\dot{y}}{y} .
$$

But in practice, in any time interval where $y$ is identically zero (or, merely, small), the observability of $x_{2}$ is singular in the sense that it is lost.

An observation $\bar{w} \in \mathcal{X}_{w}$ is said to be singular for the observation of $z$ with respect to $w$ if $\pi^{-1}(\bar{w})$ is infinite. Observations $\bar{w} \in \mathcal{X}_{w}$ which are not singular are called regular. The variable $z$ is said to be regularly observable with respect to $w$ if there is no singular observation for its observability with respect to $w$.

The best result obtained in this approach reads as follows.

Theorem 1. Let $\mathcal{X}$ be a system with variables $w, z$, and $\zeta$, and with coefficients in $\mathbf{k}$. The variable $z$ is regularly observable with respect to $w$ if $z$ is primitive over $\mathbf{k}\{w\}$.

Recall that an element $\xi$ of $\mathbf{k}\{w, z\}$ is said to be primitive over $\mathbf{k}\{w\}$ if it is a zero of a polynomial

$$
a_{d} \xi^{d}+a_{d-1} \xi^{d-1}+\cdots+a_{0}=0
$$

such that

1. the $a_{i}$ 's are in $\mathbf{k}\{w\}$,

2. the perfect differential ideal $\left\{a_{d}(w), a_{d-1}(w), \ldots, a_{0}(w)\right\}$ of $\mathbf{k}\{w\}$ is the unit ideal.

\subsection{Sensor selection}

Given a dynamic system with differential field extension

$$
\mathbf{k}\langle u, z\rangle
$$

with input $u$ and latent variable $z$ the sensor selection problem consists of the selection of sensors which endow the system with some properties. Among all such desirable properties is the basic one of observability. In this section partial answers to the following questions will be provided.

1. What is the minimal number of sensors that make the dynamics observable?

2. When the sensors are bound to measure state components, what is their minimum number? 
3. How may the observability margin be improved by selecting the sensors?

Let $y$ denote an arbitrary output of the system. By definition, an output, $y$, is componentwise algebraic over $\mathbf{k}\langle u, z\rangle$. An output makes the dynamics observable if

$$
\mathrm{d}_{\mathbf{k}\langle u, z, y\rangle}^{\circ} \mathbf{k}\langle u, y\rangle=0,
$$

that is, if each component of $z$ is algebraic over $\mathbf{k}\langle u, y\rangle$. Clearly, $y=z$ is an output which makes the system observable. Let $n$ denote the number of components of $z$, and

$$
\mathbf{I}=\left\{p \in \mathbb{N}: \exists y_{1}, y_{2}, \cdots, y_{p} \in \mathbf{k}(u, z), \mathrm{d}_{\mathbf{k}\langle u, z, y\rangle}^{\circ} \mathbf{k}\langle u, y\rangle=0\right\}
$$

The set $\mathbf{I}$ is the one of integers $p$ such that there exists an output $y$ with $p$ components which makes the system observable.

It is a nonempty ( since $n \in \mathbf{I}$ ) subset of $\mathbb{N}$. Therefore, $\mathbf{I}$ contains a smallest element which is precisely the minimal number of sensors which make the system observable.

The sensor selection problem characterizing this minimum number, $p$, of sensors is an open problem.

Later in this section it is shown that the minimum number of sensors is 1 for rational state systems, providing a partial answer to Question 1 above.

Next, about Question 2, what if the output $y$ is chosen as a subset of the components of $z$, instead of vector rational function of $u$ and $z$ ? A complete but trivial, inelegant, and computationally costy answer consists of performing the $2^{n}-1$ observability tests!

Up to the knowledge of the author there is no partial contribution to Question 3.

Back to Question 1, here is the surprising answer for the class of rational state systems.

Theorem 2. Let the state of a system $\mathcal{X}$ be given by

$$
\dot{x}=f(u, x)
$$

with a vector rational function $f$ of input $u$, state $x$, and with coefficients in a differential field $\mathbf{k}$. Let $m$ and $n$ be the respective numbers of components of $u$ and $x$. Let $\mathbf{K}$ be a differential extension field of $\mathbf{k}$. If $\mathbf{K}$ contains nonconstants then there always is a scalar output

$$
y=\sum_{i=1}^{n} \alpha_{i} x_{i}
$$

with $\alpha_{1}, \alpha_{2}, \ldots, \alpha_{n}$ in $\mathbf{K}$, which makes $x$ observable with respect to $(u, y)$. Moreover, for $y$ as in (14) to make $\mathcal{X}$ observable it is sufficient that the associated $\alpha$ 's be linearly independent over the subfield of constants of $\mathbf{K}$. 
Proof. Let $y^{[n]}$ denote the vector

$$
y^{[n]}=\left(\begin{array}{c}
y \\
\dot{y} \\
\vdots \\
y^{(n-1)}
\end{array}\right) \text {. }
$$

By Corollary 1 , for the output (14) to make $x$ observable with respect to $(u, y)$ it is necessary, and sufficient, that the Jacobian matrix of $y^{[n]}$ with respect to $x$

$$
\frac{\partial y^{[n]}}{\partial x^{\prime}}=\left(\begin{array}{c}
\frac{\partial y}{\partial x^{\prime}} \\
\frac{\partial \dot{y}}{\partial x^{\prime}} \\
\vdots \\
\frac{\partial y^{(n-1)}}{\partial x^{\prime}}
\end{array}\right)=\left(\begin{array}{cccc}
\frac{\partial y}{\partial x_{1}} & \frac{\partial y}{\partial x_{2}} & \cdots & \frac{\partial y}{\partial x_{n}} \\
\frac{\partial \dot{y}}{\partial x_{1}} & \frac{\partial \dot{y}}{\partial x_{2}} & \cdots & \frac{\partial \dot{y}}{\partial x_{n}} \\
\vdots & & & \\
\frac{\partial y^{(n-1)}}{\partial x_{1}} & \frac{\partial y^{(n-1)}}{\partial x_{2}} & \cdots & \frac{\partial y^{(n-1)}}{\partial x_{n}}
\end{array}\right)
$$

be of rank $n$ over $\mathbf{K}\langle u, y\rangle(x)$ where the complete system is considered as with coefficients in $\mathbf{K} \supseteq \mathbf{k}$.

Now $y$ may be written as

$$
y=\alpha^{\prime} x
$$

where

$$
\alpha=\left(\begin{array}{c}
\alpha_{1} \\
\vdots \\
\alpha_{n}
\end{array}\right) .
$$

The first row of $\partial y^{[n]} / \partial x^{\prime}$ is merely

$$
\frac{\partial y}{\partial x^{\prime}}=\alpha^{\prime} .
$$

The second row of the same matrix is

$$
\frac{\partial \dot{y}}{\partial x^{\prime}}=\dot{\alpha}^{\prime}+\alpha^{\prime} \frac{\partial f(u, x)}{\partial x^{\prime}} .
$$


Therefore,

$$
\operatorname{rk}_{\mathbf{k}\langle u, y\rangle(x)} \frac{\partial y^{[n]}}{\partial x^{\prime}}=\operatorname{rk}_{\mathbf{k}\langle u, y\rangle(x)}\left(\begin{array}{c}
\alpha^{\prime} \\
\dot{\alpha}^{\prime} \\
\frac{\partial \ddot{y}}{\partial x^{\prime}} \\
\vdots \\
\frac{\partial y^{(n-1)}}{\partial x^{\prime}}
\end{array}\right)
$$

by substituting the linear combination

$$
\frac{\partial \dot{y}}{\partial x^{\prime}}-\frac{\partial y}{\partial x^{\prime}} \frac{\partial f(u, x)}{\partial x^{\prime}}=\dot{\alpha}^{\prime}
$$

over $\mathbf{k}\langle u, y\rangle(x)$ of the first two rows for the second row of $\partial y^{[n]} / \partial x^{\prime}$.

More generally, by the Leibniz formula,

$$
y^{(i)}=\alpha^{(i)^{\prime}} x+\sum_{j=1}^{i}\left(\begin{array}{l}
i \\
j
\end{array}\right) \alpha^{(i-j)^{\prime}} x^{(j)},
$$

and by the fact that $x^{(j)}$ is in $\mathbf{k}\langle u\rangle(x)$ for all $j \geq 1$, it is clear that

$$
\operatorname{rk}_{\mathbf{k}\langle u, y\rangle(x)} \frac{\partial y^{[n]}}{\partial x^{\prime}}=\operatorname{rk}_{\mathbf{k}\langle u, y\rangle(x)}\left(\begin{array}{c}
\alpha^{\prime} \\
\dot{\alpha}^{\prime} \\
\ddot{\alpha}^{\prime} \\
\vdots \\
\alpha^{(n-1)}
\end{array}\right)
$$

by an immediate induction on the row's index of the Jacobian matrix.

Now note that the matrix

$$
\mathcal{W}(\alpha)=\left(\begin{array}{c}
\alpha^{\prime} \\
\dot{\alpha}^{\prime} \\
\vdots \\
\alpha^{(n-1)}
\end{array}\right)
$$

is square, and of order $n$, and does not involve neither $u$ nor $x$. Therefore it is of rank $n$ over $\mathbf{K}\langle u, y\rangle(x)$ if, and only, its determinant is nonzero.

Next note that the determinant of $\mathcal{W}(\alpha)$ is simply a differential polynomial in $\alpha_{1}, \alpha_{2}, \ldots, \alpha_{n}$ with coefficients in the field of constants of $\mathbf{K}$. Then the following theorem is used.

Theorem 3. If $G$ is a nonzero differential polynomial in $n$ indeterminates with coefficients in a differential field containing nonconstant elements then $G$ possesses a zero $\left(z_{1}, \ldots, z_{n}\right)$ over $\mathbf{K}$. 
For a proof see [13-16] for instance.

This terminates the proof of the first assertion in the theorem.

The second assertion follows from the following. The $n$ elements $\alpha_{1}, \alpha_{2}, \ldots, \alpha_{n}$ of $\mathbf{K}$ are said to be linear dependent over constants if there is a nontrivial relation

$$
c_{1} \alpha_{1}+c_{2} \alpha_{2}+\ldots+c_{n} \alpha_{n}=0
$$

with constant coefficients. It is a classical result that

Theorem 4. $\alpha_{1}, \alpha_{2}, \ldots, \alpha_{n}$ are linearly dependent over constants if, and only if, the Wronskian matrix $\mathcal{W}(\alpha)$ is singular.

For a proof see the same references [13-16] for instance. This ends the proof of the theorem.

\section{Some of the questions without partial answers}

The following is no way an exhaustive list of open problems. It is simply believed that the reader may be inspired to contribute to their solution One of the most challenging open question is actually of a foundation level.

\subsection{A foundation problem}

Observation problems are basically encountered in engineering practices where real problems often refer to real valued parameters and variables. But as the reader has already noticed the present differential algebraic geometry approach has recourse to so-called differential closures of found fields. Basically such fields are complex ones. For more details the reader may refer to section 4.1 of [6].

\subsection{Robustness}

Keeping in mind that engineering observation problems deal with systems which may be inaccurately known. The most favorable lack of information is actually that of true values of parameters or coefficients: in other words, the form and orders and dimensions of the equations are exactly known, only actual parameter values are uncertain.

The question is then how observability and other observation problems assertions behave in the presence of parameter uncertainties?

For linear systems (5) a notion of observability margin may be defined characterizing the distance of a given system to unobservable ones. This generally uses matrix tools such as singular values.

For general systems the question is clearly related to the so-called field of decisions methods for approximate systems as tacked in $[17,18]$ and many other papers. 


\subsection{Decision methods problems}

Among decision methods capable of dealing with real examples such as those one may find in biotechnology are also wanted. In this vein, there is a question with practical importance: Given an observable variable $z$ with respect to $w$, what is the minimal order of derivatives of $w$ involved in the observability of $z$ with respect to $w$ ?

\section{References}

1. Hermann, R., Krener, A.J.: Nonlinear controllability and observability. IEEE Trans. Automat. Control 22 (1977) 728-740

2. Gauthier, J.P., Kupka, I.A.K.: Deterministic Observation Theory and Applications. Cambridge University Press, Cambridge, United Kingdom (2001)

3. Pommaret, J.F.: Géométrie différentielle algébrique et théorie du contrôle. C. R. Acad. Sci. Paris Sér. I 302 (1986) 547-550

4. Fliess, M.: Quelques remarques sur les observateurs non linéaires. In: Proceedings Colloque GRETSI Traitement du Signal et des Images, GRETSI (1987) 169-172

5. Diop, S., Fliess, M.: On nonlinear observability. In Commault, C., NormandCyrot, D., Dion, J.M., Dugard, L., Fliess, M., Titli, A., Cohen, G., Benveniste, A., Landau, I.D., eds.: Proceedings of the European Control Conference, Paris, Hermès (1991) 152-157

6. Diop, S.: From the geometry to the algebra of nonlinear observability. In AnzaldoMeneses, A., Bonnard, B., Gauthier, J.P., Monroy-Perez, F., eds.: Contemporary Trends in Nonlinear Geometric Control Theory and its Applications, Singapore, World Scientific Publishing Co. (2002) 305-345

7. Pommaret, J.F.: Partial differential control theory. Volume II: Control systems. Springer Science+Business Media Dordrecht, Dordrecht, The Netherlands (2001)

8. Bejarano, F.J., Floquet, T., Perruquetti, W., Zheng, G.: Observability and detectability analysis of singular linear systems with unknown inputs. In: Proceedings of the IEEE Conference on Decision and Control, New York, IEEE Press (2011) 4005-4010

9. Diop, S., Wang, Y.: Equivalence between algebraic observability and generic local observability. In: Proceedings of the IEEE Conference on Decision and Control. Volume 3., New York, IEEE Press (1993) 2864-2865

10. Bächler, T., Gerdt, V., Lange-Hegermann, M., Robertz, D.: Thomas decomposition of algebraic and differential systems. Volume 6244 of Lect. Notes Comput. Sci., Berlin, Heidelberg, Springer (2002) 31-54

11. Lange-Hegermann, M., Robertz, D.: Thomas decompositions of parametric nonlinear control systems. Technical report (2012)

12. Sussmann, H.J.: Single-input observability of continuous-time systems. Math. Systems Theory 12 (1979) 371-393

13. Ritt, J.F.: Differential Algebra. American Mathematical Society, Providence (1950)

14. Seidenberg, A.: Some basic theorems in differential algebra (characteristic $p$, arbitrary). Trans. Amer. Math. Soc. 73 (1952) 174-190

15. Kolchin, E.R.: Differential Algebra and Algebraic Groups. Academic Press, New York (1973)

16. Kaplansky, I.: An Introduction to Differential Algebra. Second Edition. Hermann, Paris (1976) 
17. Chèze, G., Galligo, A.: From an approximate to an exact absolute polynomial factorization. J. Symbolic Comput. 41 (2006) 682-696

18. Kaltofen, E., Maye, J.P., Yang, Z., Zhi, L.: Approximate factorization of multivariate polynomials using singular value decomposition. J. Symbolic Comput. 43 (2008) 359-376 